Arq. Bras. Med. Vet. Zootec., v.70, n.2, p.633-636, 2018

\title{
Comunicação
}

[Communication]

\section{Síndrome de Eisenmenger em um gato}

[Eisenmenger syndrome in cat]

\author{
P.P.C. Costa, I.C. Barroso, M.H. Santos, L.A.R. Cabral, A.C.V. Cavalcante, F.R.N. Rodrigues, \\ J.S. Ferreira, T.M. Almeida, D.A. Viana
}

Universidade Estadual do Ceará - Fortaleza, CE

As doenças cardíacas congênitas são mais raras quando comparadas com as cardiopatias adquiridas (Bolton e Liu, 1977). Representam cerca de 5 a $15 \%$ das doenças cardíacas na espécie felina (MacDonald, 2006). Um estudo sobre a prevalência de doenças cardíacas congênitas em gatos relatou que, em 47 de 2935 de dois a seis meses de idade, ou seja, 1,6\%, foram encontrados defeitos congênitos, dentre os quais a comunicação interventricular obteve destaque com 18 casos (Schrope, 2015).

O septo ventricular é formado pela fusão do septo ventricular muscular, que cresce a partir do ápice ventricular, e da porção membranosa dorsal do septo. Se alguma parte desses componentes se desenvolver anormalmente, pode ocorrer a comunicação interventricular (Bishop, 1999). O desvio através do defeito depende do tamanho da comunicação, bem como das resistências vasculares pulmonares e sistêmicas (Scansen et al., 2015). O fluxo sanguíneo pulmonar excessivo, associado a uma grande comunicação interventricular, pode aumentar a resistência vascular pulmonar, levando à hipertensão arterial pulmonar e à inversão de desvio com hipoxemia, cianose e eritrocitose. Esse cenário clínico é denominado síndrome de Eisenmenger (SE) (Scansen et al., 2015).

Em 1897, Viktor Eisenmenger descreveu um exemplo típico da síndrome que, mais tarde, levaria seu nome, sendo descrita com um detalhado relato anatômico e clínico de um homem que apresentava dispneia e progressiva cianose, vindo a óbito com pronunciada

Recebido em 9 de janeiro de 2017

Aceito em 4 de agosto de 2017

E-mail: paula.priscila@uece.br hemoptise (MacDonald, 2006). Em conformidade, a "Síndrome de Eisenmenger" foi definida como hipertensão arterial pulmonar grave associada a um ou mais defeitos cardíacos congênitos, não tratados, com derivação bidirecional ou inversa (MacDonald, 2006). Diversos defeitos cardíacos podem resultar nessa síndrome, tais como a persistência do canal arterial, o fechamento incompleto septal atrial e/ou, ventricular.

O objetivo do presente relato foi descrever o primeiro caso de síndrome de Eisenmenger na espécie felina em território nacional.

Este relato descreve o caso de um gato, da raça Persa, de seis meses de idade, fêmea, inteira, atendida no Hospital Veterinário Sylvio Barbosa Cardoso da Universidade Estadual do Ceará. O animal apresentava histórico de dispneia, prostração, hiporexia e consequente emagrecimento progressivo havia um mês. O animal era domiciliado e sem contactantes.

Ao exame físico, observou-se apatia, epistaxe bilateral, caquexia, temperatura retal de $36,0^{\circ} \mathrm{C}$, mucosas cianóticas e hipocoradas, aumento abdominal e desidratação moderados. A auscultação torácica revelou sopro sistólico severo grau VI/VI em hemitórax direito e 180bpm. Realizou-se oxigenoterapia, num primeiro momento para estabilização, porém a paciente não resistiu e veio a óbito, sendo encaminhada para posterior exame necroscópico.

O exame post mortem apresentou macroscopicamente animal magro, com distensão abdominal acentuada, mucosa oral e oculares hipocoradas, presença de 
aproximadamente $240 \mathrm{~mL}$ de líquido amarelado na cavidade abdominal (Fig. 1). Em relação à análise da efusão cavitária, relaciona-se, nos aspectos físicos, a presença de um líquido levemente turvo, apresentando coloração amarela, ausência de coágulo e densidade igual a 1.024. Quanto às características químicas, notificaram-se $\mathrm{pH}$ neutro (7.0), concentração proteica de 3,0g/dL, sangue oculto (++-), glicose (+--) e prova de Rivalta positiva. Ressalta-se ainda, nas dosagens bioquímicas, relação albumina:globulinas de 0,43 (albumina da efusão $=0,9 \mathrm{~g} / \mathrm{dL}$ e proteína total da amostra $=3,0 \mathrm{~g} / \mathrm{dL}$ ). Referente à contagem total de células nucleadas, obteve-se uma quantidade de 400cél./ $\mu \mathrm{L}$. Na abertura da caixa torácica, evidenciou-se um coração difusamente globoso e com aumento acentuado de volume, ocupando aproximadamente $60 \%$ da cavidade torácica (Fig. 2). Observou-se solução de continuidade do septo interventricular com cerca de $2,0 \mathrm{~cm}$ de diâmetro (Fig. 3a), além de hipertrofia excêntrica ventricular esquerda e direita. Microscopicamente, as fibras cardíacas apresentavam dissociação leve tanto com congestão de capilares intramiocárdicos quanto por pequenos focos de hemorragia (Fig. 3b). Observou-se colapso pulmonar bilateral, com crepitação ausente e difusamente vermelho enegrecido (Fig. 4a) decorrente de hemorragia, edema e atelectasia (Fig. 4b). Na luz alveolar, havia grande quantidade de macrófagos livres com evidente eritrofagocitose ou acúmulo de hemossiderina (Fig. 4c), fígado com evidenciação do padrão lobular externa e internamente com áreas multifocais irregulares pardacentas, interceptadas por áreas acastanhadas (Fig. 5a), que correspondem microscopicamente a áreas de dilatação e congestão de sinusoides hepáticos associada a áreas de degeneração hidrópica (Fig. 5b). No baço, foi vista intensa hemossiderose, com presença de elementos celulares imaturos em meio a grande quantidade de megacariócitos (Fig. 6).

$\mathrm{O}$ diagnóstico da $\mathrm{SE}$ em felinos, devido à baixa incidência, é de difícil padronização. As manifestações clínicas envolvidas são aquelas também encontradas na hipertensão pulmonar grave, como taquipneia, hemoptise, estresse respiratório, intolerância a exercícios, síncope e insuficiência cardíaca congestiva (ICC) (Bach et al., 2006). O principal método de diagnóstico não invasivo da hipertensão pulmonar é a ecodopplercardiografia, a qual pode ser associada a outros exames auxiliares, como radiografias torácicas, para se avaliar a presença de sinais de ICC (Schober e Baade 2006). No entanto, a paciente veio a óbito durante o atendimento emergencial. Dessa forma, não foi possível a realização do exame supramencionado. Todavia, o diagnóstico de SE decorrente de HAP, do presente relato, foi realizado com base no quadro sintomático sugestivo de hipertensão pulmonar (Diller et al., 2013) e no exame necroscópico com as análises macroscópica e histopatológica.

A SE se desenvolve quando a hipertensão arterial pulmonar (HAP) secundária a um shunt congênito não reparado da esquerda para a direita torna-se suficientemente grave para inverter a direção do shunt (Wood, 1958), causando dessaturação periférica (Costa e Rufino, 2015). Múltiplos sistemas orgânicos são afetados como resultado da hipóxia crônica, eritrocitose e insuficiência cardíaca (Inohara et al., 2014). As complicações secundárias à cianose são: eventos isquêmicos, sangramentos, predisposição à trombose, manifestações hematológicas e infecciosas, disfunção hepática ou renal, doenças esqueléticas, disfunção ventricular direita, arritmias e morte súbita (Costa e Rufino, 2015). Inohara et al. (2014) relataram muitas dessas complicações como causas de morte. Portanto, as alterações visualizadas, macroscópicas e microscópicas, nos pulmões, no fígado, no coração e no baço estão de acordo com as complicações do quadro cianótico previsto na SE.

Em relação ao exame necroscópico, o animal apresentava distensão abdominal volumosa (ascite), com presença de $240 \mathrm{~mL}$ de líquido amarelado. Os principais diagnósticos diferenciais para as efusões, em felinos, incluem a doença inflamatória hepática, a peritonite bacteriana e a peritonite infecciosa felina (PIF). Tomando como base as características físicas, químicas e de celularidade total da efusão, o referido líquido é classificado como transudato modificado (Rizzi et al., 2007). No contexto da $\mathrm{SE}$, há a hipótese de que, devido à ICC associada e ao aumento da pressão hidrostática vascular secundária, correlacionada a uma possível hipoalbuminemia tanto por aspectos nutricionais quanto por diminuição da síntese hepática, tenha 
ocorrido a formação de ascite, e a geração, inicialmente, de um transudato puro. Nesse sentido, a permanência residual do fluido na cavidade, possivelmente, causou inflamação; por conseguinte, ocorreu o aumento de imunoglobulinas locais no líquido e transformação desse em transudato modificado. Macroscopicamente, o coração estava aumentado, de forma a ocupar aproximadamente $60 \%$ da cavidade torácica, denotando quadro de congestão com aumento das quatro câmaras. Além disso, possuía uma comunicação interventricular, como no achado de Laughlin et al. (2016), medindo $2,0 \mathrm{~cm}$ de diâmetro. Vale salientar que, no caso relatado por Eisenmenger, o paciente robusto e humano tinha uma CIV de diâmetro semelhante. Portanto, ao se comparar os dois casos, depara-se com a severidade do caso em questão. O desequilíbrio hemodinâmico severo causou aumento pressórico nos capilares intramiocárdicos, levando ao aparecimento de congestão e de pequenos focos de hemorragia. $\mathrm{Na}$ análise dos pulmões, a atelectasia e a eritrofagocitose denotam a gravidade da hipertensão pulmonar, pois, para que ambas ocorram, é necessário um aumento de volume e pressórico exorbitante, de forma a ultrapassar a capacidade dos alvéolos e dos capilares pulmonares. As alterações hepáticas, congestão de sinusoides hepáticos, associada a áreas de degeneração hidrópica, e padrão lobular, interceptadas por áreas acastanhadas, são sugestivas de quadro congestivo e hipóxico, respectivamente. A intensa hemossiderose e a grande quantidade de megacariócitos são alterações observadas no baço que corroboram o quadro de ICC e de HAP, previstos na SE.
A SE é bem relatada na medicina humana, no entanto há poucos relatos na medicina veterinária. No Brasil, até então, a doença não havia sido diagnosticada na espécie felina; no caso em questão, foi possível apenas a realização dos exames necroscópico e histopatológico, porém, consonantes com a sintomatologia do animal, foi possível, de forma assertiva, o fechamento do diagnóstico. Não obstante, faz-se necessário o estabelecimento de métodos diagnósticos in vivo para a descoberta precoce da doença, a fim de propiciar uma melhor qualidade de vida ao paciente.

$\mathrm{Na}$ paciente desta comunicação, o estágio avançado de descompensação respiratória e hemodinâmica não permitiu intervenções clínicas e terapêuticas corretivas. Ademais, a ocorrência da síndrome de Eisenmenger é incomum tanto na medicina humana quanto na veterinária e $\mathrm{o}$ presente relato descreve a ocorrência dessa complicação proveniente de uma comunicação interventricular em uma gata filhote.

Em conclusão, a SE representa a forma mais avançada da HAP, associada a defeitos congênitos, sendo uma doença complexa e multifatorial. Portanto, o presente relato mostrou aspectos importantes macroscópicos e microscópicos da SE. Dessa forma, denota-se a importância de diagnóstico e/ou do tratamento precoce de anomalias cardíacas congênitas.

Palavras-chave: defeito de septo interventricular, cardiopatia congênita, hipertensão pulmonar grave

\begin{abstract}
A 6-month-old female, $1.0 \mathrm{~kg}$, uncastrated female Persian cat was brought to the Veterinary Hospital at State University of Ceara, with a history of dyspnea, prostration, hyporexia and progressive weight loss for a month. On physical examination, systolic cardiac murmur, cyanosis and dyspnea were detected. Unfortunately, the cat died during oxygen therapy. Necropsy examination revealed an increase in cardiac silhouette and ventricular septal defect of $2 \mathrm{~cm}$ in diameter. Macroscopically the lungs were collapsed, with absent and diffusely reddish blackish crepitus, and the liver with blackish red coalescent multifocal areas, interspersed with lighter areas and lobular pattern with irregular brownish multifocal areas intercepted by brownish areas. Thus, the necropsy results together with the history and physical examination of the animal confirmed the diagnosis of Eisenmenger Syndrome, becoming the report of the first case, in a cat, in Brazil.
\end{abstract}

Keywords: defect of interventricular septum, congenital heart defect, severe pulmonary hypertension 


\section{REFERÊNCIAS}

BACH,J.F.;ROZANSKI,E.A.;MACGREGOR,J.; BETKOWSKI,J.M.;RUSH,J.E. Retrospective evaluation of sildenafil citrate as a therapy for pulmonary hypertension in dogs. J. Vet. Int. Med., v.29, p.1132-1135, 2006.

BISHOP, S.P. Embryologic development: the heart and great vessels. In: FOX, P.R.; SISSON, D.; MOISE, N.S. Textbook of canine and feline cardiology: principles and clinical practice. 2.ed. Philadelphia: W.B Saunders Company, 1999. p.3-12.

BOLTON G.R.; LIU, S.K. Congenital heart diseases of the cat. Vet. Clin. N. Am., v.7, p.341353, 1977.

COSTA, C.H.; RUFINO, R. Hipertensão Arterial pulmonar associada às cardiopatias congênitas. Pulmão RJ., v.24, p.43-46, 2015.

DILLER， G.P.; ALONSO-GONZALEZ， R.; DIMOPOULOS, K. et al. Disease targeting therapies in patients with Eisenmenger syndrome: response to treatment and long-term efficiency. Int. J. Cardiol., v.167, p.840-847, 2013.

INOHARA, T.; NIWA, K.; YAO, A. et al. Survey of the current status and management of Eisenmenger syndrome: a Japanese nationwide survey. J. Cardiol., v.63, p.286-290, 2014.
LAUGHLIN, D.S.; IALEGGIO, D.M.; TRUPKIEWICZ, J.G. et al. Eisenmenger ventricular septal defect in a Humboldt penguin (Spheniscus humboldti). J. Vet. Cardiol., v.18, p.290-295, 2016.

MACDONALD, K.A. Congenital heart diseases of puppies and kittens. Vet. Clin. N. Am. Small Anim. Pract., v.36, p.503-531, 2006.

RIZZI, T.E.; COWELL, R.L.; TYLER, R.D.; MEINKOTH, J.H. Effusions: abdominal, thoracic and pericardial. In: COWELL, R.L.; TYLER, R.D.; MEINKOTH, J.H.; DENICOLA, D.B. Diagnostic cytology and hematology of the dog and cat. 3.ed. St. Louis: Mosby, 2007. p.235- 255 .

SCANSEN, B.A.; SCHNEIDER, M.; BONAGURA, J.D. Sequential segmental classification of feline congenital heart disease. J. Vet. Cardiol., v.17, p.10-52, 2015.

SCHOBER,K.E.; BAADE,H. Doppler echocardiographic prediction of pulmonary hypertension in West Highland white terriers with chronic pulmonary disease. J. Vet. Int. Med., v.20, p.912-920, 2006.

SCHROPE, D.P. Prevalence of congenital heart disease in 76,301 mixed-breed dogs and 57,025 mixed-breed cats. J. Vet. Cardiol., v.17, p.192202, 2015.

WOOD, P. The Eisenmenger syndrome or pulmonary hypertension with reversed central shunt. Braz. Med. J., v.2, p.755-762, 1958. 